\section{$\underset{\& \text { migrations }}{\text { hommes }}$}

\section{Hommes \& migrations}

Revue française de référence sur les dynamiques

migratoires

$1324 \mid 2019$

Religion et discrimination

\title{
Géographie et intersectionnalité des actes antimusulmans en région parisienne
}

\section{Kawtar Najib}

\section{(2) OpenEdition}

\section{Journals}

Édition électronique

URL : https://journals.openedition.org/hommesmigrations/8224

DOI : 10.4000/hommesmigrations.8224

ISSN : 2262-3353

Éditeur

Musée national de l'histoire de l'immigration

Édition imprimée

Date de publication : 1 janvier 2019

Pagination : 19-26

ISBN : 978-2-919040-44-5

ISSN : $1142-852 X$

\section{Référence électronique}

Kawtar Najib, «Géographie et intersectionnalité des actes antimusulmans en région parisienne », Hommes \& migrations [En ligne], 1324 | 2019, mis en ligne le 01 janvier 2022, consulté le 08 janvier 2022. URL : http://journals.openedition.org/hommesmigrations/8224 ; DOI : https://doi.org/10.4000/ hommesmigrations.8224 


\title{
Géographie
}

et intersectionnalité

des actes antimusulmans

en région parisienne

\author{
Kawtar Najib, \\ postdoctorante Marie-Curie en géographie, université de Newcastle.
}

L'analyse socio-spatiale des discriminations touchant les populations musulmanes ou présumées comme telles dans la région parisienne se fonde sur deux perspectives complémentaires:

la géographie et les discriminations croisées. Ainsi, les femmes voilées apparaissent comme les victimes majoritaires de l'islamophobie, selon une étude qui se fonde sur l'analyse d'une seule source de données disponibles, relativement récentes (2015), collectées par le Collectif contre l'islamophobie en France. D'après cette analyse, les violences physiques et symboliques que subissent les musulmans suivraient une distribution spatiale inattendue: Paris est le département d'île-de-France le plus touché par les actes antimusulmans. Ces derniers auraient lieu au cœur de la ville, pas forcément dans les quartiers de résidence des populations qui en sont la cible. Cet article est un premier jalon d'une analyse comparée en cours sur Paris et Londres.

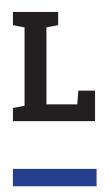

a question de la visibilité des populations musulmanes dans l'espace public a pris une grande ampleur dans les débats sociétaux contemporains. Ces débats remontent à plusieurs dizaines d'années et ont conduit à la mise en place de lois $^{2}$ qui interdisent la visibilité des signes religieux dans les espaces publics. Bien que la critique des pratiques religieuses et culturelles se doive de rester possible, cette entreprise politique ${ }^{3}$ a entraîné des dérives importantes telles que la stigmatisation grandissante des populations musulmanes, une stigmatisation qui a favorisé l'émergence
1. Cette étude s'inscrit dans le cadre d'un projet de recherche financé par la Commission européenne portant sur les espaces des actes antimusulmans dans les régions parisienne et Iondonienne (SAMA Project: Spaces of Anti-Muslim Acts in the Greater Paris and Greater London Regions). Je tiens à remercier mon superviseur Peter Hopkins pour son constant soutien ainsi que la géographe Claire Hancock pour ses premiers conseils. 2. On retrouve la loi du 15 mars 2004 (impulsée par le hijab) interdisant le port de signes religieux ostensibles, dans les établissements d'enseignements publics, et la loi du 10 octobre 2010 (impulsée par le niqab) interdisant la dissimulation du visage dans l'espace public.

3. Françoise Lorcerie (dir.), La politisation du voile en France, en Europe et dans le monde arabe, Paris, L'Harmattan, 2005. 
d'un rejet antimusulman et des actes de discrimination de plus en plus nombreux. En effet, la hausse des actes antimusulmans est attestée par les rapports de la Commission nationale consultative des droits de l'homme (CNCDH) et ceux du Collectif contre l'islamophobie en France (CCIF), tandis que la stigmatisation et les discriminations des musulmans apparaissent plus fréquentes que celles des autres groupes religieux ${ }^{4}$.

Ainsi, en étudiant l'articulation entre religion et discriminations sous le prisme de l'islam, plusieurs possibilités d'analyse apparaissent. On peut se demander, par exemple, comment l'espace public rend l'approche géographique essentielle dans les expériences de discrimination, mais aussi qui sont les principales personnes concernées par ces discriminations. Dans ce sens, cet article propose une analyse socio-spatiale des discriminations touchant les populations musulmanes ou présumées musulmanes dans la région parisienne. Il permet, d'une part, de présenter les espaces et les lieux où se sont produits les actes antimusulmans et, d'autre part, de mieux identifier et décrire les victimes. Il s'agit d'apporter une contribution géographique aux recherches portant sur l'islamophobie, tout en mettant l'accent sur l'intersectionnalité du phénomène. En effet, la discrimination antimusulmane ne peut s'analyser sans faire de liens avec d'autres types de discriminations telles que le sexisme, le racisme, l'âgisme et le classisme. Ces intersections s'inscrivent également dans des organisations spatiales spécifiques que l'outil cartographique permet de dégager.

\section{Contribution géographique aux recherches sur l'islamophobie}

Si les travaux scientifiques portant sur l'islamophobie se sont multipliés ces dernières années ${ }^{5}$, peu d'entre eux se focalisent sur sa dimension spatiale. Pourtant, l'espace se trouve au centre des préoccupations suscitées par le phénomène du rejet antimusulman. Par exemple, les prières de rue ont rendu visibles les Musulmans et provoqué des paniques morales dans certaines villes françaises, tout comme la visibilité des jeunes filles voilées dans les établissements d'enseignement public qui a conduit à promulguer des lois d'interdiction des signes religieux dans les lieux publics. Il en est de même pour les femmes portant le niqab qui ne peuvent plus se déplacer dans les espaces publics sans prendre le risque de recevoir une amende forfaitaire. Ainsi, la géographie - et surtout la géographie sociale qui place l'individu au centre des rapports qu'il entretient avec son environnement - apparaît comme une approche privilégiée dans l'étude des discriminations religieuses. Il existe quelques géographes qui étudient ce phénomène et leurs travaux abordent cette question d'abord par le biais de la justice spatiale, du racisme, de l'identité et du féminisme géographique ${ }^{6}$. Cette contribution cherche quant à elle à observer la distribution géographique de l'islamophobie, c'est-à-dire à identifier les espaces où se sont produits les actes antimusulmans dans la région parisienne.

\section{(2) Si les travaux scientifiques portant sur l'islamophobie se sont multipliés ces dernières années, peu d'entre eux se focalisent sur sa dimension spatiale.}

Pour ce faire, il s'agit de collecter et d'analyser des données géoréférencées. Nous avons recueilli les indications géographiques des actes antimusulmans auprès des principaux organismes qui les recensent:

\footnotetext{
4. Rapport de l'Observatoire européen des phénomènes racistes et xénophobes (EUMC), « Les Musulmans au sein de l'Union européenne : discrimination et islamophobie », 2006; Commission nationale consultative des droits de l'homme (CNCDH), La lutte contre le racisme, l'antisémitisme et la xénophobie, Rapport de la Commission nationale consultative des droits de l'homme de 2013, Paris, La documentation française, 2014 ; Collectif contre l'islamophobie en France (CCIF), Rapport annuel sur l'islamophobie en France en 2015, 2016 ; Tell Mama, Geography of Anti-Muslim Hatred in 2015, Annual Report 2015, 2016, pp. 38-51.
}

5. Fred Halliday, «Islamophobia Reconsidered », in Ethnic and Racial Studies, vol. 22, n 5, 1999, pp. 892-902; Amir Saeed, "Media, racism and islamophobia: The representation of Islam and Muslims in the media », in Sociology Compass, vol. 1, $n^{\circ} 2$, 2007, pp. 443-462; Nasar Meer, Tariq Modood, « Refutations of racism in the "Muslim question" », in Patterns of Prejudice, vol. 43, n 3-4, 2009, pp. 335-354 ; Chris Allen, Islamophobia, Farnham/ Burlington, Ashgate, 2010.

6. Kevin M. Dunn, Natascha Klocker, Tanya Salabay, "Contemporary racism and Islamophobia in Australia », in Ethnicities, vol. 7, n 4, 2007, pp. 564-589; Claire Dwyer, « Veiled meanings: Young british muslim women and the negotiation of differences », in Gender, Place \& Culture, vol. 6, n 1, 1999, pp. 5-26; Claire Hancock, " "The Republic is lived with an uncovered face" (and a skirt): (un)dressing French citizens », in Gender, Place \& Culture, vol. 22, n 7, 2015, p. 1023-1040; Peter Hopkins, Gurchathen Sanghera, Katherine Botterill, Rowena Arshad, "Young non-muslims face Islamophobia too », in The Conversation, 2 mars 2017. 
le ministère de l'Intérieur et le CCIF. Ce dernier possède, à l'échelon individuel, une base de données plus riche puisqu'il comptabilise les déclarations qui lui sont rapportées (après une vérification des éléments matériels les corroborant), contrairement au ministère de l'Intérieur qui ne prend en compte que les actes ayant abouti à un dépôt de plainte. Les analyses qui suivent s'appuient essentiellement sur la base de données du CCIF et plus précisément sur les données de 2015 enregistrées dans la région parisienne, la région qui comptabilise le plus fort taux d'actes antimusulmans ${ }^{8}$. En effet, le CCIF a recueilli 905 actes islamophobes dont $43 \%$ uniquement en île-de-France (suivie par la région Auvergne-RhôneAlpes qui enregistre $17 \%$ des actes, puis par la région Provence-Alpes-Côte-d'Azur qui en recense 8 \%). Ces actes islamophobes n'ont cessé d'augmenter jusqu'en 2016, passant de 691 actes en 2013 à 764 en 2014, et donc à 905 en 2015, soit une hausse de respectivement plus de $10 \%$ et $18 \%$ pour les périodes 2013-2014 et 2014-2015. On distingue en 2015, parmi ces actes, $65 \%$ de cas de discriminations, $17 \%$ de discours islamophobes, $7 \%$ de dégradations-profanations, $6 \%$ d'agressions physiques et $5 \%$ d'agressions verbales ${ }^{9}$. En effet, avec les nombreuses attaques terroristes et le contexte d'état d'urgence, l'année 2015 enregistre ce chiffre sans précédent de 905 actes qui se différencie fortement des statistiques ministérielles. Le ministère de l'Intérieur compte 429 actes antimusulmans en 2015 et observe, lui aussi, une forte augmentation des actes par rapport à l'année précédente; les chiffres auraient triplé entre 2014 et $2015^{10}$.

\section{Les espaces et les lieux de l'islamophobie dans la région parisienne}

Les espaces où se sont produits les actes antimusulmans peuvent faire référence soit à des espaces géographiques, soit à des lieux spécifiques (tels que le lieu de travail, une institution publique, les transports en commun, etc.). Selon les faits enregistrés en 2015, 64 \% des actes se sont déroulés dans une institution publique (que ce soit une mairie, une école ou encore un hôpital). Ainsi, les services publics sont les premiers lieux de discrimination. En fait, certains fonctionnaires pensent avoir le droit d'étendre le champ d'application de la loi de 2004 à l'ensemble des usagers des services publics. Le reste des discriminations émane de personnes physiques ou morales: on compte $2 \%$ de personnes physiques (qui peuvent être des passants dans la rue) et $34 \%$ de personnes morales (qui peuvent être des patrons d'entreprise par exemple). On peut donc remarquer que l'écrasante majorité des discriminations (98\%) s'inscrit dans un cadre institutionnel ou professionnel, contrairement à ce que l'on peut observer dans d'autres pays, notamment en Grande-Bretagne, où les actes antimusulmans ont d'abord lieu dans les transports publics ${ }^{11}$. Ce constat peut nous amener à nous interroger sur les mécanismes de fonctionnement de l'islamophobie en France. L'impact de la loi du 15 mars 2004 peut être considéré comme un processus descendant qui émanerait d'abord des institutions étatiques et non des individus eux-mêmes.

Les actes islamophobes ont donc lieu davantage dans le centre parisien que dans les autres départements de la banlieue parisienne (voir figure 1). C'est pourquoi je propose de montrer, dans la figure 2, une carte présentant la distribution spatiale des actes recensés dans Paris intra-muros. L'ensemble des vingt arrondissements est concerné par le phénomène à des degrés différents. Aucune dissymétrie vraiment nette n'apparaît entre le Nord et le Sud, l'Est et l'Ouest ou le centre et la périphérie, contrairement à l'échelle du Grand Paris ${ }^{12}$ et de l'île-de-France où l'on peut

7. Les actes antimusulmans sont définis d'après le CCIF comme l'ensemble des actes de discrimination ou de violence contre des individus ou des institutions en raison de leur appartenance réelle ou supposée à l'islam, en s'appuyant sur les remontées directes des victimes. Alors que le ministère de l'Intérieur définit les actes antimusulmans comme l'ensemble des actions (homicides, attentats, incendies, dégradations, violences et voies de fait) et des menaces (propos, gestes menaçants et démonstrations injurieuses, inscriptions, tracts et courriers) qui font l'objet d'une plainte ou d'une main courante auprès de la police. Voir Commission nationale consultative des droits de l'homme (CNCDH), « Rapport sur la lutte contre le racisme, l'antisémitisme et la xénophobie », Rapport de la Commission nationale consultative des droits de l'homme, Les essentiels du Rapport sur la lutte contre le racisme 2017, 2017, 39 p.

8. Cet article prend en compte des données issue du rapport du CCIF de 2015. II existe cependant des données du CCIF plus récentes (2017) qui n'ont pas été prises en compte dans cette étude.

9. Collectif contre l'islamophobie en France (CCIF), 2016, op. cit.

10. Cécile Chambraud, « Les actes antimusulmans en forte baisse en 2016 », in Le Monde, 1er février 2017.

11. Tell Mama, op. cit. ; Muslim Engagement and Development (MEND), Anti-Muslim Hate Crime 2014, Rapport 2014, ODIHR/ OSCE, 2015, 82 p

12. Le Grand Paris compte les départements de Paris (75), des Hauts-de-Seine (92), de Seine-Saint-Denis (93) et du Val-de-Marne (94). 


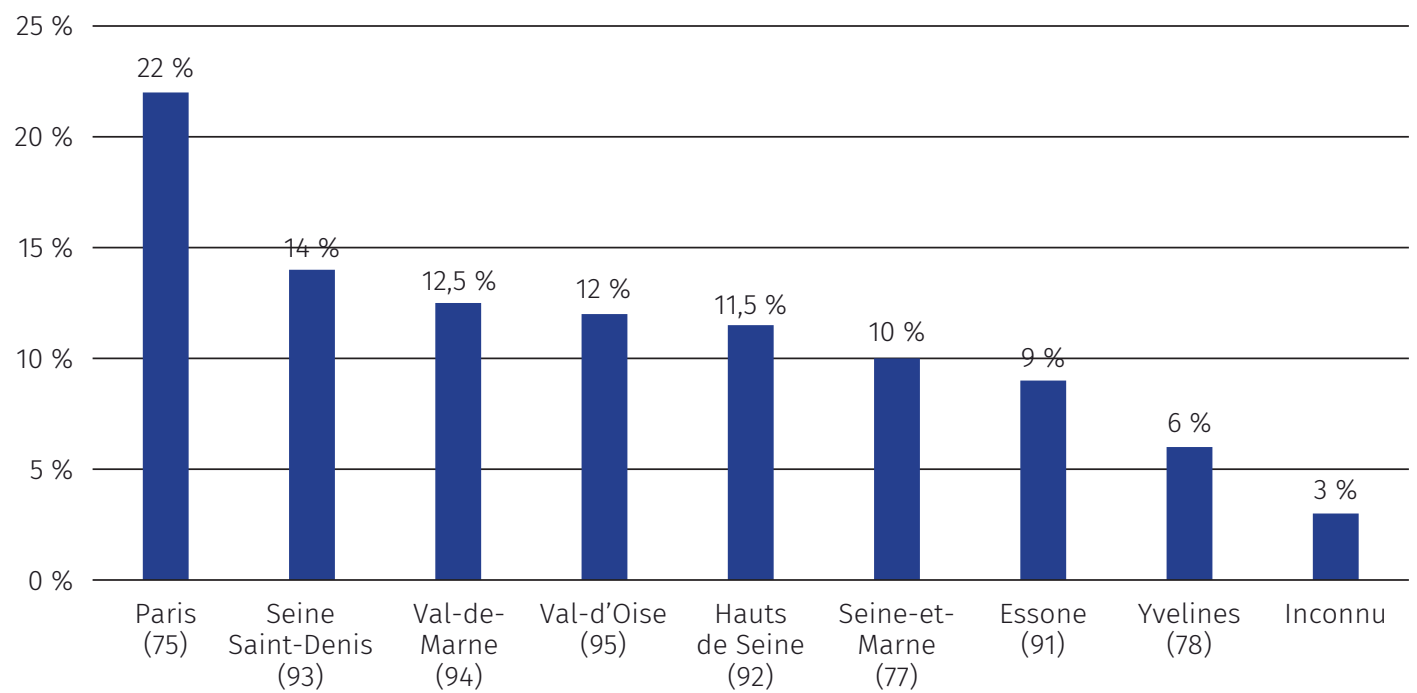

Figure 1 : Les actes antimusulmans en île-de-France.

Source : Données du Collectif contre l'islamophobie en France (CCIF), 2015.

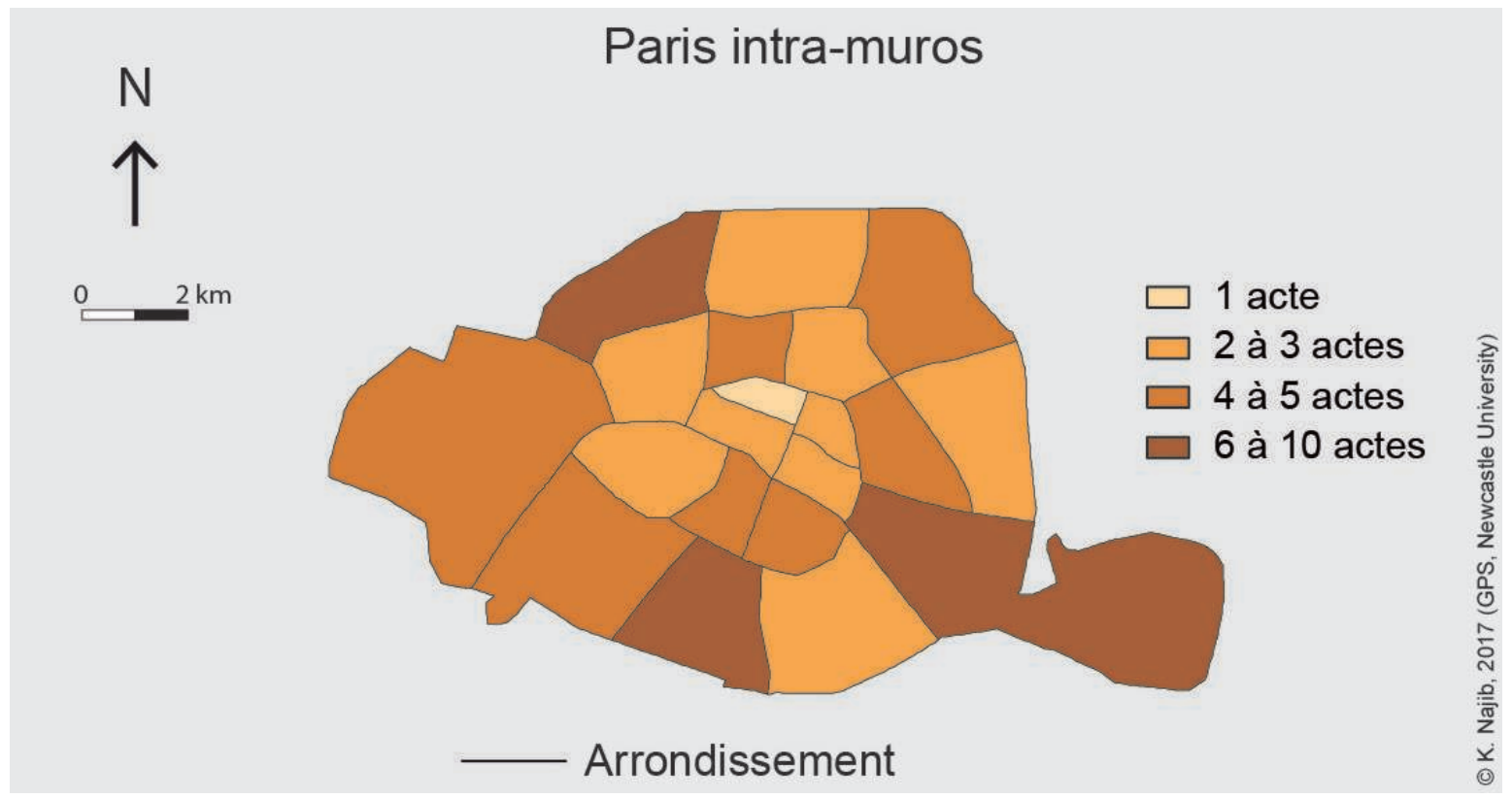

Figure 2 : Localisation des actes antimusulmans à Paris intra-muros.

Source: Données du Collectif contre l'islamophobie en France (CCIF), 2015.

observer que le centre parisien est le plus touché ${ }^{13}$. De plus, avec le détail de la position géographique, on observe que les actes antimusulmans n'ont pas forcément lieu dans les quartiers populaires du centre parisien comme par exemple les quartiers de la Goutte-d'Or ou de Belleville. Cependant, on s'aperçoit tout de même que certains quartiers visés par la politique de la ville dans les $10^{\mathrm{e}}, 11^{\mathrm{e}}$ et $19^{\mathrm{e}}$ arrondissements, mais aussi dans le Nord du $17^{\mathrm{e}}$ arrondissement comptabilisent des actes islamophobes non négligeables. En outre, les $17^{\mathrm{e}}, 12^{\mathrm{e}}$ et $14^{\mathrm{e}}$ arrondissements correspondent aux arrondissements qui enregistrent les chiffres les plus importants. Ils enregistrent respectivement 6,7 et 10 actes antimusulmans. Le

13. Kawtar Najib, Peter Hopkins, « De Londres à Paris, regards sur l'islamophobie», in The Conversation, 17 avril 2018. 
Tableau 1 : Localisation des actes antimusulmans dans des ZUS ou non dans le Grand Paris

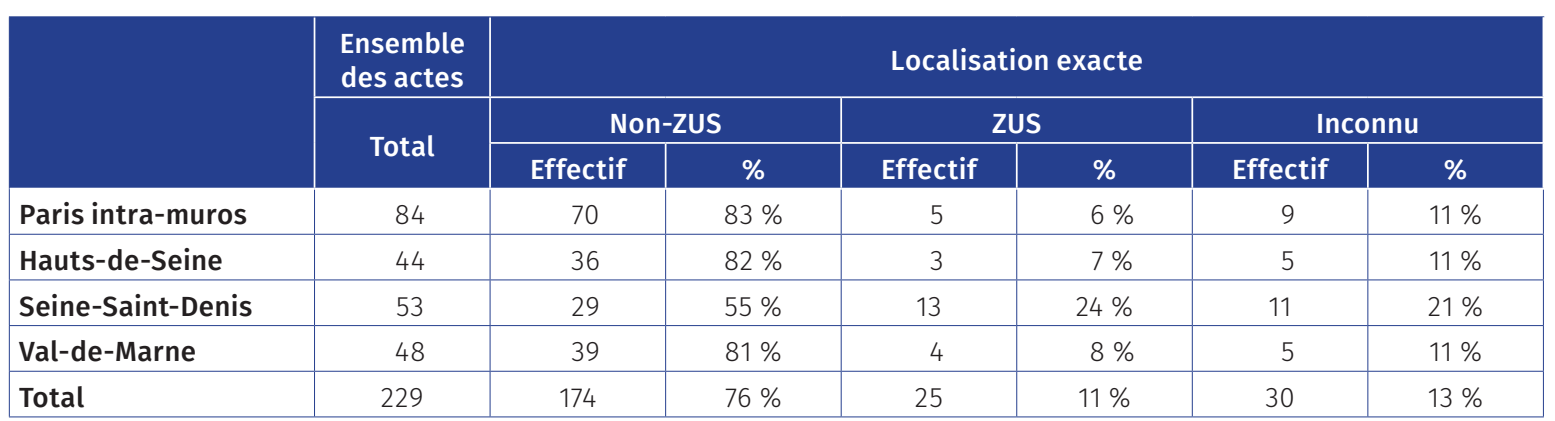

Source : Données du Collectif contre l'islamophobie en France (CCIF), 2015 et données du Comité interministériel des villes, 2010.

$14^{e}$ arrondissement compte donc le plus grand nombre d'actes islamophobes, mais il convient ici de préciser que cinq personnes (ayant chacune légitimement contacté le CCIF) ont vécu un acte de discrimination dans un seul et même endroit, c'est-à-dire plus précisément dans une situation professionnelle commune. Au final, on observe qu'à l'échelle du Grand Paris ou de l'île-de-France les espaces de l'islamophobie répondent à des modèles d'organisation urbaine de type centre-périphérie opposant la ville-centre et sa banlieue (les actes antimusulmans seraient donc plus nombreux au centre qu'en périphérie). Ce modèle aurait également été observé dans d'autres villes européennes, notamment à Malmö en Suède ${ }^{14}$.

Au-delà du centre parisien, suivant la localisation précise des actes antimusulmans ayant eu lieu dans le Grand Paris, on note, d'après le tableau 1, que seuls $11 \%$ des actes se sont produits dans une zone urbaine sensible (ZUS), alors que la région île-deFrance comprend le plus grand nombre de ZUS en France et que la population vivant en ZUS dans le Grand Paris est seulement de $11 \%$ d'après le recensement de la population de 2006 de l'Institut national de la statistique et des études économiques (Insee $)^{15}$.

La majorité des actes antimusulmans (76\%) s'observe donc dans des zones non-classifiées par la politique de la ville. Les travaux de recherche montrent que la majorité les populations musulmanes se localisent dans la banlieue parisienne ${ }^{16}$ et sans doute dans les zones populaires et sensibles car ces populations sont souvent associées, en France, aux classes populaires et à l'immigration ${ }^{17}$. Dans ce sens, on sait que les ZUS comptent un grand nombre de familles précaires et d'étrangers, et que la répartition spatiale, plus précisément, des étrangers hors Union européenne obéit à des modèles urbains qui décrivent une structure en mosaïque où les pôles de concentration correspondent aux ZUS ${ }^{18}$. On peut donc considérer que les populations musulmanes y habitent en grande majorité ${ }^{19}$ puisqu'elles vivent principalement dans les quartiers populaires ayant une forte population immigrée. Finalement, ces résultats montrent que les lieux où se sont produits les actes antimusulmans ne correspondent pas nécessairement aux espaces habités, en grande majorité, par les populations musulmanes. Ce constat va dans le sens des travaux de l'urbaniste Carina Listerborn ${ }^{20}$ qui montre bien, avec l'exemple des femmes voilées, que les espaces de l'islamophobie peuvent révéler une certaine opposition, voire une contradiction, entre des espaces généralement bien considérés et valorisés socialement, mais où ces personnes discriminées se sentent vulnérables, et des espaces généralement stigmatisés et craints par le plus grand nombre où elles se sentent à l'aise.

14. Carina Listerborn, « Geographies of the veil: violent encounters in urban public spaces in Malmö, Sweden », in Social \& Cultural Geography, vol. 16, n 1, 2015, pp. 95-115.

15. Corinne Chevalier, «La population des zones urbaines sensibles ", in Insee première, n 1328, 2010, 4 p. ; Yoann Musiedlak, "Les ZUS franciliennes : un paysage contrasté », in Insee Île-de-France, n 356, mai 2011, 9 p.

16. Hervé Vieillard-Baron, «De la difficulté à cerner les territoires du religieux : le cas de l'islam en France », in Annales de Géographie, vol. 113, n 640, 2004, pp. 563-587.

17. Jonathan Laurence, Justin Vaïsse, Intégrer l'islam : la France et ses musulmans, enjeux et réussites, Paris, Odile Jacob, 2007; Patrick Simon, Vincent Tiberj, Sécularisation ou regain religieux: la religion des immigrés et de leurs descendants, Documents de travail n 196, Série Trajectoires et origines, Ined, 2013, 46 p.

18. Jean-Louis Pan Ké Shon, «Portrait statistique des zones urbaines sensibles », in Informations sociales, $n^{\circ} 141,2007$, pp. 24-32; Harris Selod, «La mixité sociale : le point de vue des sciences économiques », in Informations sociales, $n^{\circ} 125,2005$, pp. 28-35.

19. Jonathan Laurence, Justin Vaïsse, op. cit.

20. Carina Listerborn, op. cit. 


\section{La perspective intersectionnelle de l'islamophobie}

L'intersectionnalité, également étudiée en géographie et notamment en géographie féministe ${ }^{21}$, représente une autre piste d'analyse de l'islamophobie. Les discriminations opérées au nom de l'islam peuvent s'expliquer par le fait que cette religion est, dans chacune de ses manifestations, interprétée comme un problème public en France ${ }^{22}$, mais aussi et surtout par le fait qu'elle porte en elle d'autres indicateurs, tout aussi délicats, liés à l'immigration, au passé colonial, aux classes populaires, à l'oppression de la femme et à un danger terroriste ${ }^{23}$. Cet enchevêtrement de représentations négatives contribue à la multiplication des actes de discrimination ou de violence à l'encontre d'institutions ou d'individus en raison de leur appartenance réelle ou supposée à l'islam ${ }^{24}$.

\section{(1) Finalement, ces résultats montrent que} les lieux où se sont produits les actes antimusulmans ne correspondent pas nécessairement aux espaces habités, en grande majorité, par les populations musulmanes.

Plus précisément, il convient de s'intéresser, ici aux discriminations visant les individus et ainsi de croiser leurs critères sociodémographiques et ethniques afin de mieux mettre en évidence cette intersectionnalité. Il convient notamment de montrer que l'islam est devenu une religion «racialisée» comme elle le fut un temps durant les périodes de colonisation ${ }^{25}$. L'islamophobie peut apparaître comme un processus de racialisation d'individus présumés musulmans, un processus qui consiste à essentialiser sous ce seul attribut religieux un ensemble d'individus des plus variés ${ }^{26}$. Parfois, des personnes non-musulmanes (telles que des Sikhs ou des athées de culture arabo-musulmane ou encore des femmes qui portent un voile en raison d'une maladie ou d'une certaine mode vestimentaire) sont également victimes d'islamophobie ${ }^{27}$. Ainsi, l'idée est de prendre en compte la perspective intersectionnelle de l'islamophobie puisque les discriminations qui en découlent peuvent cacher d'autres formes de discriminations liées au genre, à l'âge, à l'origine ethnique ou au statut socioprofessionnel de l'individu ${ }^{28}$. Ces autres discriminations ne possèdent en effet aucune justification «acceptable» contrairement à l'islamophobie qui peut être perçue comme la simple critique d'une religion ${ }^{29}$. Cela dit, de nombreux auteurs disent que l'hostilité à l'encontre de l'islam et le rejet des musulmans sont intrinsèquement corrélés ${ }^{30}$, un rejet qui, lui-même, peut conduire à des formes de violences.

21. Gillian Rose, Feminism and Geography, Cambridge, Polity Press, 1993 ; Audrey Kobayashi, Linda Peake, « Unnatural discourse: "race" and gender in geography », in Gender, Place \& Culture, vol. 1, n² 2, 1994, pp. 225-243; Leslie Kern, Beverley Mullings, « Neoliberalism, urban insecurity and urban violence: exploring the gender dimensions », in Linda Peake, Martina Rieker (dir.), Rethinking Feminist Interventions into the Urban, New York, Routledge, 2013, pp. 23-40 ; Eleonore Kofman, Annie Phizacklea, Parvati Raghuram, Rosemary Sales, Gender and International Migration in Europe: Employment, Welfare and Politics, Londres, Routledge, 2000 ; Audrey Kobayashi, « Neoclassical urban theory and the study of racism in geography », in Urban Geography, vol. $35, n^{\circ} 5,2014$, pp. 645-656

22. Françoise Lorcerie, op. cit. ; Abdellali Hajjat, Marwan Mohammed, Islamophobie. Comment les élites françaises construisent le "problème musulman », Paris, La Découverte, 2013.

23. Lila Abu-Lughod, «Do Muslim women really need saving? Anthropological reflections on cultural relativism and its others », in American Anthropologist, vol. 104, n 3, 2002, pp. 783-790 Gholam Khiabany, Milly Williamson, « Veiled bodies - naked racism: Culture, politics and race in the Sun », in Race and Class, vol. 50, n², 2008, pp. 69-88; Peter Hopkins, The Issue of Masculine Identities for British Muslims After 9/71: A Social Analysis Lewiston/New York, Edwin Mellen Press, 2008 ; Lynn A. Staeheli, Caroline R. Nagel, « Rethinking security: Perspectives from Arab-American and British Arab activists », in Antipode, $n^{\circ} 40$, $n^{\circ}$ 5, 2008, pp. 780-801; Patrick Simon, Vincent Tiberj, op. cit. 24. Observatoire européen des phénomènes racistes et xénophobes (EUMC), op. cit. ; Brian Klug, «Islamophobia: A concept comes of ages », in Ethnicities, vol. 12, n 5, 2012 pp. 665-681 ; Nasar Meer, Tariq Modood, op. cit.

25. Olivier Le Cour Grandmaison, De l'indigénat, Paris, La Découverte, 2010

26. Kevin M. Dunn, Natascha Klocker, Tanya Salabay, op. cit. ; Nasar Meer, Tariq Modood, op. cit. ; Chris Allen, op. cit.

27. Peter Hopkins, Gurchathen Sanghera, Katherine Botterill, Rowena Arshad, op. cit. ; Jennifer Hyndman, Deborah Cowen, Natalie Oswin, Rupal Oza, Audrey Kobayashi, Jasbir K. Puar, «Terrorist assemblages: Homonationalism in queer times », in Social \& Cultural Geography, vol. 11, n 4, 2010, pp. 399-409.

28. Vincent Geisser, La nouvelle islamophobie, Paris, La Découverte, 2003 ; Carina Listerborn, op. cit. ; Tariq Modood, «Introduction: The politics of multiculturalism in the New Europe », in Tariq Modood, Prina J. Werbner (dir.), The Politics of Multiculturalisme in the New Europe, Londres, Zed Books, 1997 pp. 1-26.

29. Collectif contre l'islamophobie en France (CCIF), Rapport annuel sur l'islamophobie en France en 2013, 2014, 22 p.

30. Nasar Meer, Tariq Modood, op. cit. ; Runnymede Trust, Islamophobia: A Challenge for Us All, Londres, Runnymede Trust, 1997 ; Brian Klug, op. cit. 


\section{Les femmes, premières victimes des actes antimusulmans}

Ainsi, en analysant les critères sociodémographiques des discriminés à partir des données de 2015 du CCIF, on sait que les actes antimusulmans touchent d'abord les femmes, et de surcroît les femmes voilées. Les femmes représentent $74 \%$ des victimes et les femmes voilées au moins $65 \%$ des femmes victimes. En prenant en compte uniquement les discriminations, elles sont $88 \%$ et les femmes voilées au moins $77 \%$, que ce soit celles qui portent un hijab (86\%), un jilbeb (7\%), un turban ( $4 \%$ ), un simple bandana (2\%) ou encore un niqab (1\%). Les agressions physiques concernent aussi principalement les femmes (82\%) et les femmes voilées (au moins $80 \%$ ). Dans des domaines bien précis, elles peuvent même représenter la totalité des victimes: c'est le cas du domaine académique et des formations professionnelles ${ }^{31}$. La place de la femme voilée est devenue un problème au moment où son intégration sociale l'a rendue visible dans l'espace social, c'est-à-dire au moment même où on a commencé à la voir à l'école, à l'université, sur le marché du travail. Il est donc intéressant de constater, dans ce contexte, comment le corps et la tenue vestimentaire des femmes constituent un enjeu dans les politiques d'intégration, et notamment dans la construction de la citoyenneté et de l'identité nationale ${ }^{32}$. Ainsi, l'islamophobie peut se présenter aussi comme une forme de sexisme ${ }^{33}$ puisque les femmes sont davantage ciblées que les hommes. Elles sont, en effet, plus affectées par les discriminations car elles sont à la fois des cibles plus visibles (en portant le voile) et plus fragiles (lorsqu'elles sont seules).

Concernant l'âge des victimes, on observe que les jeunes sont très touchés par les discriminations; ils représentent 33 \% des victimes en 2015. L'islamophobie semble donc plus affecter les jeunes ${ }^{34}$ que les moins jeunes. Les personnes âgées pratiquant l'islam, susceptibles d'être des étrangers ou des immigrés, font moins l'objet d'islamophobie car leurs pratiques seraient davantage associées aux coutumes de leur pays d'origine, contrairement aux jeunes qui sont susceptibles de vivre un véritable regain religieux ${ }^{35}$, parfois en lien avec leurs expériences des discriminations ${ }^{36}$. Ces étrangers ou immigrés plus âgés seraient également moins demandeurs d'un accès à une quelconque égalité et seraient également moins réactifs aux discriminations antimusulmanes. L'étude montre que les jeunes ayant grandi en France, demandant l'égalité de l'accès aux mêmes droits que tous, se sentent plus exclus lorsqu'ils portent un signe d'appartenance à la religion musulmane. Face à cette exclusion, certains d'entre eux ${ }^{37}$ réagissent en créant de nouveaux modes d'engagement éthique et politique ${ }^{38}$.

Quant à la catégorie socioprofessionnelle, on retrouve parmi les victimes une forte proportion de personnes faisant partie de la catégorie, c'est-à-dire «sans activité professionnelle». Ils sont $35 \%$ et peuvent faire référence soit à des femmes au foyer, soit à des non-réponses. Les chômeurs représentent 8 \% des victimes et les employés $12 \%$. On remarque, enfin, qu'aucune victime ne fait partie de la catégorie des "cadres et professions intellectuelles supérieures». Cette question de la classe sociale permet de préciser quelque peu le profil socio-économique des populations musulmanes qui subissent des discriminations. Ces populations sont plutôt précaires, mais la différence de traitement, selon qu'on fasse partie - ou plus précisément qu'on soit perçu comme faisant partie d'une classe aisée ou d'une classe défavorisée, a pu être observée, notamment par le biais d'entretiens qualitatifs que j'ai menés auprès de femmes voilées victimes d'islamophobie et vivant à Paris. Certaines m'ont confié qu'elles passaient plus inaperçues dans certains espaces touristiques parisiens luxueux, comme par exemple la célèbre avenue des ChampsÉlysées, car on les prenait très souvent pour de riches touristes venant du Qatar ou d'Arabie saoudite.

31. Collectif contre l'islamophobie en France (CCIF), 2016, op. cit. 32. Mayanthi L. Fernando, The Republic Unsettled: Mus/m French and the Contradictins of Secularism, Durham, Duke University Press, 2014 ; Joan Wallach Scott, The Politics of the Veil, Princeton, The Princeton University Press, 2007.

33. Carina Listerborn, op. cit.

34. Ingrid Ramberg, L'islamophobie et ses conséquences pour les jeunes, Strasbourg, éd. du Conseil de l'Europe, 2005.

35. Jocelyne Cesari, Musulmans et républicains : les jeunes, l'islam et la France, Bruxelles, Complexe, 1998 ; Patrick Simon, Vincent Tiberj, op. cit.

36. Karen Phalet, Mérove Gijsberts, Louk Hagendoorn, "Migration and religion: Testing the secularisation thesis among Turkish and Moroccan Muslims in the Netherlands 1998-2005 », in Migration and Integration, vol. 48, 2008, pp. 412-436 ; Scott Poynting, Victoria Mason, «The resistible rise of Islamophobia: Anti-Muslim racism in the UK and Australia before 11 September 2001 », in Journal of Sociology, vol. 43, n 1, 2007, pp. 61-86.

37. Raphaël Liogier, Le mythe de l'islamisation. Essai sur une obsession collective, Paris, Seuil, 2012.

38. Gerdien Jonker, Valérie Amiraux, Politics of Visibility. Young Muslims in European Public Spaces, Bielefeld, Transcript Verlag, 2006 ; Marco Martiniello, Patrick Simon, «Les enjeux de la catégorisation », in Revue européenne des migrations internationales, vol. 21, n² 2, 2005 ; Mayanthi L. Fernando, op. cit. 
Enfin, si le CCIF ne possède pas de données vraiment détaillées sur l'origine ethnique des victimes, nous savons qu'à l'échelle nationale $81 \%$ des victimes sont des musulmans dits «de naissance » contre $12 \%$ qui se déclarent converties à l'islam. De plus, on sait que la population musulmane française est en grande majorité issue de l'immigration des Trente glorieuses ${ }^{39}$. On peut donc supposer que ces musulmans de naissance peuvent être soit des étrangers, soit des Français d'origine étrangère venant d'Afrique du Nord, d'Afrique subsaharienne ou encore de Turquie. Ainsi, les populations musulmanes qui se font discriminer en fonction de leur appartenance religieuse peuvent également l'être par rapport à leur origine ethnique. Dans ce sens, les rapports du CCIF montrent bien qu'un certain nombre d'actes et de discours islamophobes renvoient aux origines étrangères des victimes et notamment à leur «arabité $e^{40}$ ». Pour plus de précisions, nous avons identifié la consonance des noms et prénoms des victimes vivant en région parisienne, et on peut noter que $81 \%$ des victimes portent un nom ou prénom à consonance arabe, contre $10 \%$ française ou européenne et $6 \%$ africaine subsaharienne (le reste correspondant à des dossiers sans nom ou difficilement identifiable).

\section{Un profil type de la victime discriminée}

Par conséquent, cette analyse permet, malgré la petite taille du panel, d'esquisser un profil type, à savoir une femme en l'occurrence voilée, étudiante et d'origine arabe. Ainsi, les discriminations touchent les populations les plus fragiles, c'est-à-dire les femmes, les personnes précaires et d'origine étrangère. L'étude de l'intersectionnalité du phénomène de l'islamophobie décrit finalement une domination générale des plus faibles déjà connue dans nos sociétés contemporaines. En outre, ce profil type de la victime discriminée peut nous aider à analyser celui du discriminant, comme l'ont dépeint certains rapports sur les crimes de haine antimusulmane en Grande-Bretagne ${ }^{41}$, à savoir un homme blanc britannique âgé de 25 à 59 ans. Cela dit, dans son rapport de 2010, le CCIF expliquait qu'une agression sur deux était l'œuvre d'une femme ${ }^{42}$; ce que montrent également les données de 2015 sur la région parisienne. Parmi l'ensemble des actes enregistrés, on compte - quand on dispose de l'information - autant de femmes ( $42 \%$ ) que d'hommes ( $42 \%$ ). Cette question du profil type du discriminant permet de se demander si l'islamophobie renvoie d'abord à une domination masculine (l'étude montre des hommes qui s'attaquent à des femmes très souvent seules ou accompagnées d'enfants en bas âge) ou si, à l'inverse, elle souligne une histoire de "féministes à deux vitesses» (des femmes qui s'attaquent à d'autres femmes au nom de l'émancipation des femmes).

\section{Conclusion}

Le véritable enjeu d'une vision géographique portant sur l'articulation entre religion et discriminations repose sur le fait que l'espace apparaît d'abord comme un opérateur de catégorisation des groupes. En effet, les populations musulmanes sont généralement désignées comme «Autres ${ }^{43} 》$. Ce processus d'altérisation, basé d'abord sur une vision dualiste entre «eux» et «nous», soulève une autre opposition dans sa dimension spatiale: celle entre «ici» et «là-bas ${ }^{44}$ ». En définitive, la discrimination antimusulmane n'a d'autres effets que de marginaliser une population déjà fragile ${ }^{45}$. Ainsi, il ne s'agit pas d'un simple contact malheureux entre un discriminant et un discriminé, mais bien de comprendre ce que signifie être musulman en France et quelle place leur est accordée, c'est-à-dire de mieux comprendre pourquoi cette minorité apparaît comme une menace à l'ordre urbain et à la culture nationale occidentale ${ }^{46}$.

39. Patrick Simon, Vincent Tiberj, op. cit.

40. Collectif contre l'islamophobie en France (CCIF), Rapport annuel sur l'islamophobie en France en 2010, 2011, 33 p. ; Collectif contre l'islamophobie en France (CCIF), Etre musulmane aujourd'hui en France: les femmes, premières victimes de l'islamophobie, Rapport CCIF 2014/2015, 2015, 46 p.

41. Nigel Copsey, Jack Dack, Mark Littler, Matthew Feldman, Anti-Muslim Hate Crime and the Far Right, Centre for Fascist, Anti-Fascist and Post-Fascist Studies, Teesside, Teesside University Press, 2013 ; Tell Mama, op. cit.

42. Collectif contre l'islamophobie en France (CCIF), 2011, op. cit

43. Christine Delphy, Classer, dominer. Qui sont les «autres »? Paris, La fabrique éditions, 2008 ; Françoise Lorcerie, Vincent Geisser, Muslims in Marseille. At Home in Europe Project, New York, Open Society Foundations Report, 2011 ; Amir Saeed, op. cit.

44. John Clayton, «Thinking spatially: towards an everyday understanding of inter-ethnic », in Social \& Cultural Geography, vol. 10, n 4, 2009, pp. 481-498; Claire Hancock, «"The Republic is lived with an uncovered face" (and a skirt): (un)dressing French citizens $»$, op. cit.

45. Joan Wallach Scott, op. cit.

46. Claire Hancock, «La justice au risque de la différence : faire une "juste place" à l'Autre », in Annales de géographie, n 665-666, 2009, pp. 61-75; Annelies Moors, " The Dutch and the face-veil: The politics of discomfort», in Social Anthropology, vol. 17, $n^{\circ} 4$, 2009, pp. 393-408 ; Raphaël Liogier, op. cit. ; Carina Listerborn, op. cit. 